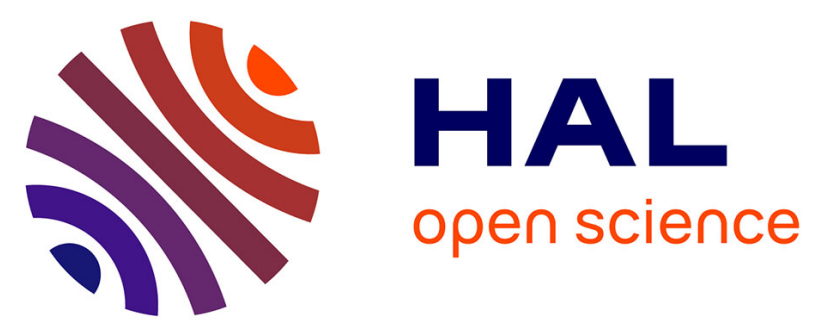

\title{
Feasibility and impact of the implementation of a clinical scale-based sedation-analgesia protocol in severe burn patients undergoing mechanical ventilation. A before-after bi-center study
}

Raphaël Cinotti, Noémie Besnard, Luc Desmedt, Ronan Le Floch, Pierre Perrot, Farid Bekara, Kada Klouche, R. Larcher, Pierre-Joachim Mahé, Denis Frasca, et al.

\section{- To cite this version:}

Raphaël Cinotti, Noémie Besnard, Luc Desmedt, Ronan Le Floch, Pierre Perrot, et al.. Feasibility and impact of the implementation of a clinical scale-based sedation-analgesia protocol in severe burn patients undergoing mechanical ventilation. A before-after bi-center study. Burns, In press, 10.1016/j.burns.2020.02.009 . hal-02507680

\author{
HAL Id: hal-02507680 \\ https://hal.science/hal-02507680
}

Submitted on 1 Jul 2020

HAL is a multi-disciplinary open access archive for the deposit and dissemination of scientific research documents, whether they are published or not. The documents may come from teaching and research institutions in France or abroad, or from public or private research centers.
L'archive ouverte pluridisciplinaire HAL, est destinée au dépôt et à la diffusion de documents scientifiques de niveau recherche, publiés ou non, émanant des établissements d'enseignement et de recherche français ou étrangers, des laboratoires publics ou privés. 


\title{
Feasibility and impact of the implementation of a clinical scale-based sedation-analgesia protocol in severe burn patients undergoing mechanical ventilation. A before-after bi-center study
}

\author{
Raphaël Cinotti ${ }^{a, *}$, Noémie Besnard ${ }^{b}$, Luc Desmedt $^{c}$, Ronan Le Floch ${ }^{c}$,
} Pierre Perrot $^{d}$, Farid Bekara ${ }^{e}$, Kada Klouche ${ }^{b, f}$, Romaric Larcher ${ }^{b}$, Pierre-Joachim Mahéc ${ }^{c}$ Denis Frasca ${ }^{g, h}$, Karim Asehnoune, ${ }^{c, i}$, Boris Jung ${ }^{b}$, Antoine Roquilly ${ }^{c, i}$

\footnotetext{
${ }^{a}$ Department of Anesthesia and Critical Care, Hôpital Guillaume et René Laennec, University Hospital of Nantes, Boulevard Jacques Monod, Saint-Herblain 44800, France

${ }^{\mathrm{b}}$ Medical Intensive Care Unit, Hôpital Lapeyronie, Montpellier University and MontpellierTeaching Hospital, 191, Avenue du Doyen Gaston Giraud, MontpellierCedex 5, Montpellier, 34295, France

c Anesthesia and Critical Care, University Hospital of Nantes, Hôtel Dieu, 1 place Alexis Ricordeau, Nantes 44093, France ${ }^{\mathrm{d}}$ Department of Plastic and Burn Surgery, University Hospital of Nantes, Hôtel Dieu, 1 place Alexis Ricordeau, Nantes 44093, France

e Department of Plastic and Burn Surgery, Montpellier University and Montpellier Teaching Hospital, Hôpital Lapeyronie 191, Avenue du Doyen Gaston Giraud, Montpellier Cedex 5, Montpellier 34295, France

${ }^{\mathrm{f}}$ INSERM U1046, CNRS UMR9214, Hôpital Lapeyronie 191, Avenue du Doyen Gaston Giraud, MontpellierCedex 5, Université deMontpellier, Montpellier 34295, France

${ }^{g}$ Department of Anesthesia and Critical Care, Centre Hospitalo-Universitaire, University Hospital of Poitiers, 2 rue de la Milétrie Poitiers 86021, France

$\mathrm{h}$ INSERM SPHERE U1246 «MethodS for Patients-centered outcomes and HEalth REsearch», UFR des sciences pharmaceutiques, University of Nantes, University of Tours, 22 boulevard Benoni-Goullin, Nantes 44200, France ${ }^{i}$ Laboratoire UPRES EA 3826 «Thérapeutiques cliniques et expérimentales des infections». University hospital of Nantes, 22 boulevard Benoni-Goullin, Nantes 44200, France
}

Keywords:

Sedation

Analgesia

Mechanical ventilation

Weaning

\begin{abstract}
A B S T R A C T
Background: Severe burn patients undergo prolonged administration of sedatives and analgesics for burn care. There are currently no guidelines for the dose adaptation of sedation-analgesia in severe burn patients.

Methods: We performed a before-after 2-center study to demonstrate the feasibility and efficacy of a sedation-analgesia scale-based protocol in severely burned patients receiving $\geq 24 \mathrm{~h}$ of invasive mechanical ventilation. Before the intervention, continuous infusion of hypnotic and morphine derivatives was continued. During the Intervention phase, general anesthesia was relayed from day 1 by RASS/BPS-titrated continuous infusion of hypnotic and morphine derivatives and with short half-life drugs adminstered
\end{abstract}

\footnotetext{
* Corresponding author at: Department of Anesthesia and Critical Care, Hôpital Guillaume et René Laennec, University Hospital of Nantes, Boulevard Jacques Monod, Saint-Herblain 44800, France.

E-mail address: raphael.cinotti@chu-nantes.fr (R. Cinotti).
} 
for daily burn dressings. The primary outcome was the duration of invasive mechanical ventilation in the ICU.

Results: Eighty-seven (46.2\%) patients were included in the Control phase and 101 $(53.7 \%)$ in the Intervention phase. The median burned cutaneous surface was $20 \%$ [11\%-38\%] and median ABSI was 7 [5-9]. The durations of hypnotic and opioid infusions were not statistically different between the 2 phases ( 8 days [2-24] vs. 6 days [2-17] $(\mathrm{P}=0.3)$ and 17 days [4-32] vs. 8 days [3-23] $(\mathrm{P}=0.06)$, respectively). The duration of mechanical ventilation was 14 days [3-29] in the Control phase and 7 days [2-24] in the Intervention phase $(\mathrm{P}=0.7)$. When taking into account the competition between mortality and weaning from mechanical ventilation, we found no significant difference between the 2 phases (Gray test, $P=0.4$ ). The time-series analysis showed no difference for the duration of mechanical ventilation in the Intervention phase $(\mathrm{P}=0.6)$. Eighteen $(20.7 \%)$ patients died in the Control phase, and $18(18 \%)$ in the Intervention phase $(\mathrm{P}=0.6)$.

Conclusion: Scale-based lightening of continuous sedation-analgesia with repeated short general anesthesia for dressing is feasible in severe burn patients but failed to demonstrate a decrease in the duration of invasive mechanical ventilation.

\section{Introduction}

In the United Kingdom, approximately 29,000 patients were hospitalized for burn treatment between 2003 and 2007 [1]. Among them between $4 \%$ and $22 \%$ were transferred to a specialized critical care unit (ICU) with an overall mortality of around 3\% [1]. Prolonged general anesthesia is frequently used in severe burn patients in order to control pain secondary to burns, perform skin graft surgery and daily dressing changes [2]. This heavy sedation results in prolonged invasive mechanical ventilation. However, it is now well established in the general ICU population that prolonged infusion of hypnotics and opioids has major adverse effects [3]. Several randomized clinical trials have demonstrated that dose adaptation of sedatives and pain killers to validate clinical scales decreases the duration of mechanical ventilation and enhances the outcomes of general ICU patients [4]. However, burn patients have always been excluded from these studies. There are therefore no data regarding the feasibility and the effects of scale-based lightening of sedation and analgesia in severe burn patients. Pain management at the initial phase of a burn is challenging since pain killers are needed continuously and periodically during procedures such as dressings and the prevention of chronic pain is also a priority objective. Evidence-based guidelines on this topic are therefore urgently needed.

We hypothesized that lightening sedation and analgesia was feasible and would reduce the adverse effects of prolonged sedation in burn patients. We have therefore implemented a scale-based, nurse-controlled sedationanalgesia protocol based on validated clinical scales and associated with general anesthesia with short half-life drugs to control procedural pain in critical burn patients on prolonged mechanical ventilation. We performed a beforeafter study in 2 centers which implemented this protocol and studied its effects on the duration of mechanical ventilation.

\section{Methods}

\subsection{Ethics and approvals}

We conducted a before-after study in 2 centers (Surgical and Burn ICU, Hôtel-Dieu, University Hospital of Nantes, France and the Medical ICU, University Hospital of Montpellier, France) between January 1, 2014, and November 1, 2018 in patients admitted for burns and receiving at least $24 \mathrm{~h}$ of mechanical ventilation. The protocol was approved by the Société Française d'Anesthésie-Réanimation Institutional Review Board (CERAR, IRB 00010254-2016-040). Since this study evaluated the effects of the implementation of a standard of care, consent was waived. Oral and written information were nonetheless provided to patients and next-of-kin.

\subsection{Study population}

Consecutive patients with a burn injury and $\geq 15$ years old were included in this study. Patients were eligible in case of an expected mechanical ventilation duration $\geq 24 \mathrm{~h}$. Patients were excluded if they presented a Glasgow Coma Score $\leq 13$ during initial care, anoxic brain damage secondary to carbon monoxide intoxication, pregnancy or breast feeding. Patients with withdrawal of life-sustaining therapies in the $24 \mathrm{~h}$ after admission were not eligible.

\subsection{Study design}

The Control phase consisted of all consecutive burned patients admitted to our ICUs 2 years before the educational program began (from January 1, 2014 to January 1, 2016 in the main center and from May 1, 2015 to May 1, 2016 in the partner center). Since burn patients in the ICU traditionally undergo large doses of sedative and opioid agents, the aim of our study was to evaluate the effects of a scale-based sedation-analgesia protocol. The protocol was elaborated by one center (the main center) to provide a bedside, scale-based (Richmond 
Assessment Sedation Scale (RASS) for hypnotics [3-5] and Behavior Pain Scale (BPS) for opioids [6,7]) and a nursecontrolled sedation and analgesia protocol. The intervention was introduced over a 3-month period (from October to December 2015 in the main center and from February to April 2016 in the partner center) during which no data were collected. The Intervention phase included all consecutive patients with burns admitted to the participating ICUs during a 2-year period (2016-2018).

\subsection{General burn care}

Our units are 2 regional reference burn centers. Initial surgical burn care includes early wound debridement and closure [8]. Burn care often involves excision which can be performed either at the initial phase or in the first days following initial resuscitation if a damage-control strategy is chosen. After excision and initial care, skin grafts are performed and harvested from an unburned area of the body. Skin graft surgery can be performed several times in case of a large burn surface area or graft failure until the entire burned area is healed. In case of extensive burns with little donor zone, temporary wound coverage can be performed with allograft or xenograft [9].

Initial care also includes adequate resuscitation with vascular expansion according to the Parkland rule adapted to urine output (0.5 and $\left.1 \mathrm{~mL} . \mathrm{kg}^{-1} \cdot \mathrm{h}^{-1}\right)$ [10], blood transfusion, endo-tracheal intubation in case of burned airway and/or a burned cutaneous surface $\geq 20 \%$, respiratory or circulatory failure. We performed early tracheostomy (in the first week following admission) in patients with a total burned cutaneous surface $\geq 40 \%$ and patients with severe face/neck burn injury with significant airway impairment assessed with a fiberoptic evaluation and cuff-leak test, $24 \mathrm{~h}$ after hospitalization.

\subsection{Control phase}

During the Control phase, the analgesia-sedation protocol associated a continuous infusion of midazolam $\left(0.1-0.2 \mathrm{mg} \cdot \mathrm{kg}^{-1} \cdot \mathrm{h}^{-1}\right)$ and oxycodone $\left(0.05-0.1 \mathrm{mg} \cdot \mathrm{kg}^{-1} \cdot \mathrm{h}^{-1}\right)$. Ketamine and gabapentine were not used during this phase. The daily doses of midazolam and sufentanil were left to the attending physician's discretion. Sedation and analgesia were discontinued i) in the absence of major complication(s) (septic shock, acute respiratory distress syndrome), ii) when surgical skin grafts were completed, iii) when the remaining burned area was considered small enough to allow spontaneous breathing. During procedural analgesia (skin dressings), remifentanil was added [11]. During this phase, sedation and analgesia were not managed by nurses.

\subsection{Interphase}

During this period, a nurse-controlled scale-based analgesia and sedation protocol was implemented in both units over a 3-month period. The aim of the training was to explain to nurses how to adapt the infusion of hypnotic and analgesic drugs according to validated scales (RASS and BPS).

\subsection{Intervention phase}

In the main center, the protocol was implemented after January 1, 2016 and in the partner center after May 1, 2016. The main center elaborated the protocol which was applied in the main and partner centers. Briefly, analgesia-sedation was adapted with RASS and BPS targets set by the junior or senior physicians. The RASS/BPS targets were adapted daily to clinical evolution. Nurses could titrate the infusion of hypnotic and opioid so the patient would remain within the specified target (usually RASS and BPS were set at $0 /$ 1 and $<5$ respectively). The initial prescription also associated ketamine $\left(0.5 \mathrm{mg} \cdot \mathrm{kg}^{-1} \cdot \mathrm{h}^{-1}\right)$ in the first five days after admission. During painful burn care and dressing changes, RASS and BPS targets were set at $-5 /-4$ and $<4$ respectively, along with i) a bolus of $1 \mathrm{mg} \cdot \mathrm{kg}^{-1}$ and $1 \mathrm{mg} \cdot \mathrm{kg}^{-1} \cdot \mathrm{h}^{-1}$ infusion of ketamine, ii) a continuous infusion of remifentanil and propofol if indicated. When burn care and dressing were resolved, ketamine and remifentanil were stopped and RASS/BPS were set at their previous targets. After ICU admission, pregabaline $(75 \mathrm{mg}$ twice a day) was also administered in order to prevent secondary hyperalgesia. During the intervention phase, no awakening trials or sedation holidays were performed. The entire protocol and its implementation are detailed in the Supplemental Digital Content. In the Intervention phase, there was no other modification in the general care of the burn patients (surgery, critical care).

\subsection{MV weaning protocol}

After sedation discontinuation, patients were checked at least once a day according to predefined weaning criteria [12]: stable cardiovascular status (heart rate 140 beats/min or less, systolic blood pressure $90-160 \mathrm{mmHg}$, and minimal or absence of catecholamine), adequate oxygenation (oxygen saturation measured by pulse oximetry $\geq 90 \%$, fractional inspired oxygen tension $\leq 40 \%$, positive end-expiratory pressure $\leq 8 \mathrm{~cm} \mathrm{H}_{2} \mathrm{O}$, respiratory rate $\leq 35$ breaths $/ \mathrm{min}$ ), $\mathrm{PaCO}_{2} \leq 50 \mathrm{mmHg}$, core temperature $\leq 38.5^{\circ} \mathrm{C}$. Spontaneous breathing trial was systematically performed in all patients with a 30-min T-tube trial. Failure of the weaning test was defined as the development within $30 \mathrm{~min}$ of any of the following criteria: respiratory rate $\geq 35$ breaths/min with increased accessory muscle activity, $\mathrm{SpO}_{2} \leq 90 \%$, heart rate greater than 140 beats $/ \mathrm{min}$, systolic blood pressure $\leq 90 \mathrm{mmHg}$ or $\geq 180 \mathrm{mmHg}$, major dyspnea or agitation, or GCS $\leq 8$ or less. Once the patient displayed weaning criteria, MV was stopped and the patient was extubated. In our unit, this active protocol was associated with a reduction in the duration of $\mathrm{MV}$ in brain-injured patients [13]. This standard of care was maintained during both periods.

\subsection{Data collection}

We collected demographic data (age, gender, body mass index, medical history, psychiatric disorders, previous medication), burn characteristics and management (\% of burned body area, smoke aspiration, mechanism of burn injury, airway burn injury, Abbreviated Burn Severity Index, number of surgical procedures for skin grafts), in-ICU management 
(tracheostomy, ventilator acquired pneumonia, acute respiratory distress syndrome, duration of mechanical ventilation, duration of catecholamine infusion, withdrawal of life sustaining therapies), analgesia and sedation management (duration of hypnotic drugs, duration of opioid drugs, monitoring of RASS/BPS and modification of hypnotics/ opioids in the first $48 \mathrm{~h}$ after ICU admission), hospital length of stay, in-hospital mortality and mortality at day 90 .

\subsection{Primary outcome}

The primary outcome was the in-ICU duration of invasive mechanical ventilation. Invasive mechanical ventilation was defined as the duration between the first day of mechanical ventilation and the day of successful weaning. Successful weaning was considered when mechanical ventilation was successfully removed during a $48 \mathrm{~h}$ period. Whenever mechanical ventilation was started again, for a respiratory issue for instance, the duration of invasive mechanical ventilation was increased accordingly. For example, a few hours of unexpected mechanical ventilation for atelectasis was considered as one day of MV. However, elective surgery for burn care receiving general anesthesia and mechanical ventilation for a few hours was not considered for the primary outcome.

\subsection{Secondary outcomes}

The secondary outcomes were the rate of application of the protocol in the $48 \mathrm{~h}$ after admission, the duration of hypnotic and opioid drugs, the occurrence of ventilator acquired pneumonia, acute respiratory distress syndrome, catecholamine infusion, the number of surgical procedures for skin grafts, withdrawal of life sustaining therapies, the number of ventilatory-free days at day 90, in-ICU and mortality at day 90 . We also studied extubation success between both phases in the following sub-groups: center, patients with a burned body surface $\geq 20 \%$, patients with tracheostomy, patients $\geq 70$ years old.

\subsection{Statistical analysis}

Continuous data are expressed as mean ( \pm standard deviation) or median [quantile] and compared with Student's t-test or Mann-Whitney test whenever appropriate. Nominal data are expressed as $\mathrm{N}(\%)$ and compared with the Chi2 or Fisher test whenever appropriate. Owing to the before-after design of the study, we elaborated an interrupted time-series analysis. We studied the segmented linear regression in the control and intervention periods and compared the regression coefficient of the linear trend between both periods. The aggregated value was the mean of the duration of mechanical ventilation per each semester. First, we looked for autocorrelated errors of the aggregated duration of mechanical ventilation score in the overall cohort with an ARIMA model. Auto-correlated errors were checked with a graphical analysis and the Ljung-Box test. Second, we analyzed the effect of time on the aggregated value with an ARIMA model. We then elaborated a segmented linear regression in the Control and the Intervention phases and compared the $\beta$ regression coefficient of the linear regression between the Control and the Intervention phases $[14,15]$.

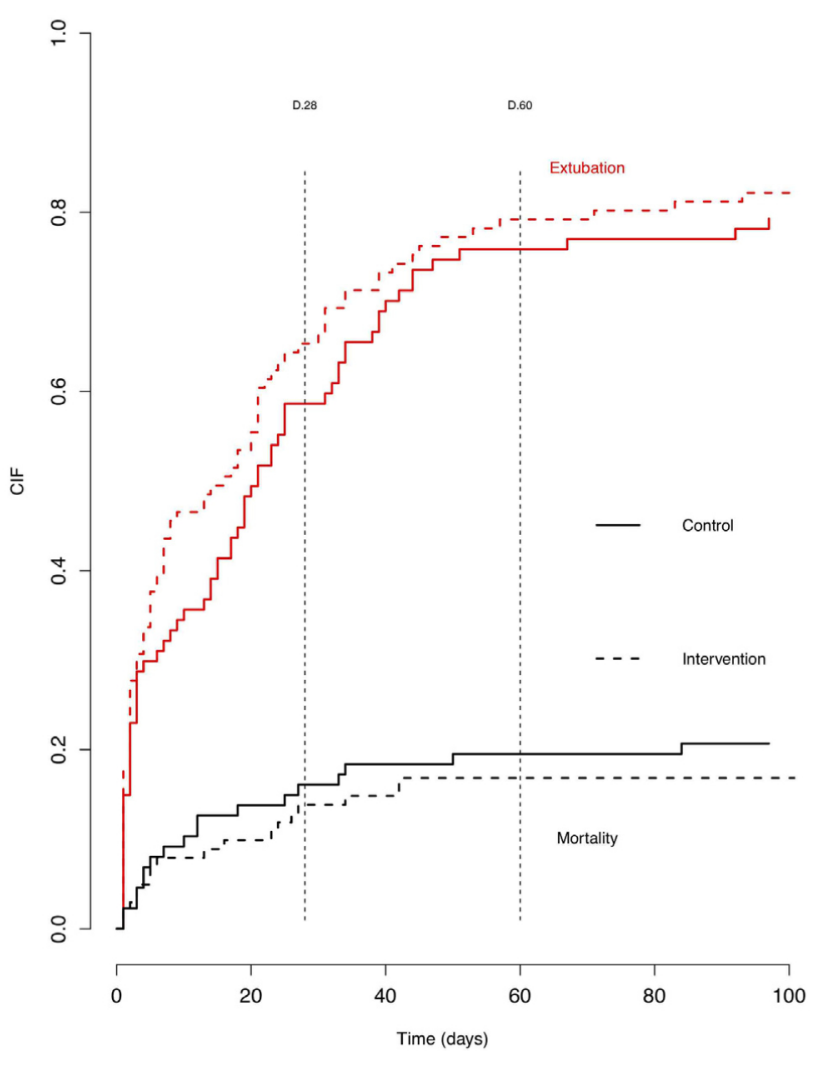

Fig. 1-Aalen-Johanssen estimator regarding the competition between mortality and mechanical ventilation. Non-significant trend towards a shorter duration of mechanical ventilation between the 2 phases, Gray test, $P=0.4$.CIF: Cumulative Incidence Function.

Given the competition between mortality and the duration of mechanical ventilation, we studied the duration of mechanical ventilation with an Aalen-Johanssen estimator between the 2 periods [16]. Finally, we explored extubation success between the two centers. Using a Log-rank test with mortality as a competitive event in the overall cohort (Control and Intervention phases), we calculated the odds ratio of extubation success in various subgroups (center, patients with a burned cutaneous surface $\geq 20 \%$, patients with tracheostomy, patients $\geq 70$ years old). All statistical tests were two-sided. A p value $<0.05$ was considered statistically significant. Statistical analyses were performed with $\mathrm{R}$ Studio ${ }^{\mathbb{R}}$ version 1.0.136, with the etm package (The "R" Foundation for Statistical Computing, Vienna, Austria).Fig. 1

\section{Results}

We included 188 patients in the overall population, 87 (46.2\%) in the Control phase and 101 (53.7\%) in the Intervention phase. The median burned cutaneous surface was 18\% [10-39] before and $20 \%$ [12-35] after the intervention $(\mathrm{P}=0.3)$, while the median ABSI was 7 [5-9] before and 7 [5-9] after $(\mathrm{P}=0.9)$. There were 146 patients $(77.7 \%)$ included in the main center. There were no statistically significant differences regarding the rates of airway burn injury ( $83.9 \%$ vs. $78.2 \%, \mathrm{P}=0.3)$ or the 


\begin{tabular}{llll}
\hline Main center & $73(83.9)$ & $73(72.3)$ & 0.06 \\
Age & $48[33-61]$ & $49[35-63]$ & 0.9 \\
Gender Male/Female & $59(67.8) / 28(32.2)$ & $70(69.3) / 31(30.7)$ & 0.8 \\
BMI & $24[22-29]$ & $25[23-30]$ & $36(35.6)$ \\
Tobacco consumption & $30(34.5)$ & $20(19.8)$ & 0.2 \\
Alcohol consumption & $19(21.8)$ & $33(32.7)$ & 0.9 \\
History of psychiatric disease & $32(36.8)$ & $18(17.8)$ & 0.9 \\
Hypertension & $11(12.6)$ & & \\
Chronic medication & & $34(33.7)$ & 0.6 \\
Benzodiazepine & $23(26.4)$ & $17(16.8)$ & $19(18.8)$ \\
Neuroleptics & $16(18.4)$ & & 0.3 \\
Antidepressant drug & $20(23)$ & $20[12-35]$ & \\
Burn characteristics & & $7[5-9]$ & $79(78.2)$ \\
\% of burned body area & $18[10-39]$ & $21(20.8)$ \\
ABSI & $7[5-8]$ & $87(86.1)$ & 0.8 \\
Airway burn injury & $73(83.9)$ & $47[40-52]$ \\
Smoke aspiration & $16(18.4)$ & 0.9 \\
Burn by fire & $81(93.1)$ & 0.9 \\
SAPS II & $42[37-50]$ & 0.3 \\
\hline
\end{tabular}

Legend: SAPS: Simplified Acute Physiology Score. BMI: Body Mass Index. ABSI: Abbreviated Burn Severity Index.

mechanism of burn (fire injury in 81 (93.1\%) patients vs. 87 (86.1\%) patients, $\mathrm{P}=0.9)$. Baseline characteristics are displayed in Table 1.

\subsection{Primary outcome}

The median duration of invasive MV was 14 [3-29] days in the Control phase and 7 [2-24] days in the Intervention phase $(\mathrm{P}=0.7)$. Owing to the competitive risk between mortality and the duration of mechanical ventilation, we took into consideration the competitive risk between death and weaning from invasive mechanical ventilation with the Aalen-Johanssen model [16], the duration of invasive ventilation was not different between the two periods $(P=0.4)$.

Given the study design and in order to investigate a potential secular trend in the evolution of the duration of invasive mechanical ventilation, we performed a timeinterrupted time-series analysis. There were no errors of the aggregated duration of mechanical ventilation score in the overall cohort with an ARIMA model or and the Ljung-Box test, implying a lack of seasonal trend. In the segmented linear regression between the Control and the Intervention phases, there was a non-significant decrease of the aggregated value of the mechanical ventilation $(\mathrm{P}=0.6)$ (Fig. 2).

\subsection{Secondary outcomes}

As for protocol management in the $48 \mathrm{~h}$ after admission, the RASS and the BPS scales were prescribed respectively in 70 $(96 \%)$ and 68 (93\%) patients in the main center. During the intervention, the RASS and BPS were recorded 10 [6-16] and 11 [6-16] times respectively. The doses of hypnotics and analgesics were adapted $2[1-4]$ and $2[0-4]$ times respectively. In the partner center, the RASS and the BPS scales were prescribed respectively in $28(100 \%)$ and $26(93 \%)$ patients. During the intervention, the RASS and BPS were recorded 15
[13-16] and 14 [13-15] times respectively. The doses of hypnotics and analgesics were adapted 5 [4-7] and 3 [2-4] times respectively.

In both centers, the duration of hypnotic infusion was 8 days [2-24] in the Control phase and 6 days [2-17] in the Intervention phase $(\mathrm{P}=0.3)$. The duration of opioid infusion was 17 days [4-32] before vs. 8 days [3-23] after the intervention $(\mathrm{P}=0.06)$.

As for exploratory analyses, we investigated the effects of the intervention in pre-specified subgroups. The odds ratio of spontaneously breathing patients during the intervention phase was $1.3 \mathrm{IC}_{95}[0.9-1.9]$ in the main center, $1.2 \mathrm{IC}_{95}[0.5-2.6]$ in the partner center, $1.1 \mathrm{IC}_{95}[0.7-1.8]$ in severe burn patients (burned cutaneous surface $\geq 20 \%$ ) and $0.9 \mathrm{IC}_{95}$ [0.5-1.5] in patients with tracheostomies (Fig. 3).

We found no differences regarding the occurrence of ventilator-associated pneumonia (49 (56.3\%) vs. 47 (47\%), $\mathrm{P}=0.2$ ), the number of surgeries for skin graft (2 [1-4] vs. 2 [1-4], $\mathrm{P}=0.9)$. In the Control phase, $18(20.7 \%)$ patients died in the ICU and $18(18 \%)$ died in the Intervention phase $(\mathrm{P}=0.6)$. Secondary outcomes are provided in the Table 2.

\section{Discussion}

In our before-after 2-center study, the implementation of a scale-based, nurse-controlled sedation-analgesia protocol was feasible but was not associated with a reduction in the duration of invasive MV in severe burn patients.

In recent years, the management of sedation and analgesia with clinical scales such as the RASS or BPS [4] has emerged in order to prevent the side effects of prolonged drug infusion. The titration of hypnotics and opioids with these scales could decrease the duration of mechanical ventilation in the general ICU population [6] and has shown to be beneficial in decreasing mortality [17]. It is now therefore recommended to monitor the level of analgesia and sedation in ICU patients [3]. However, 


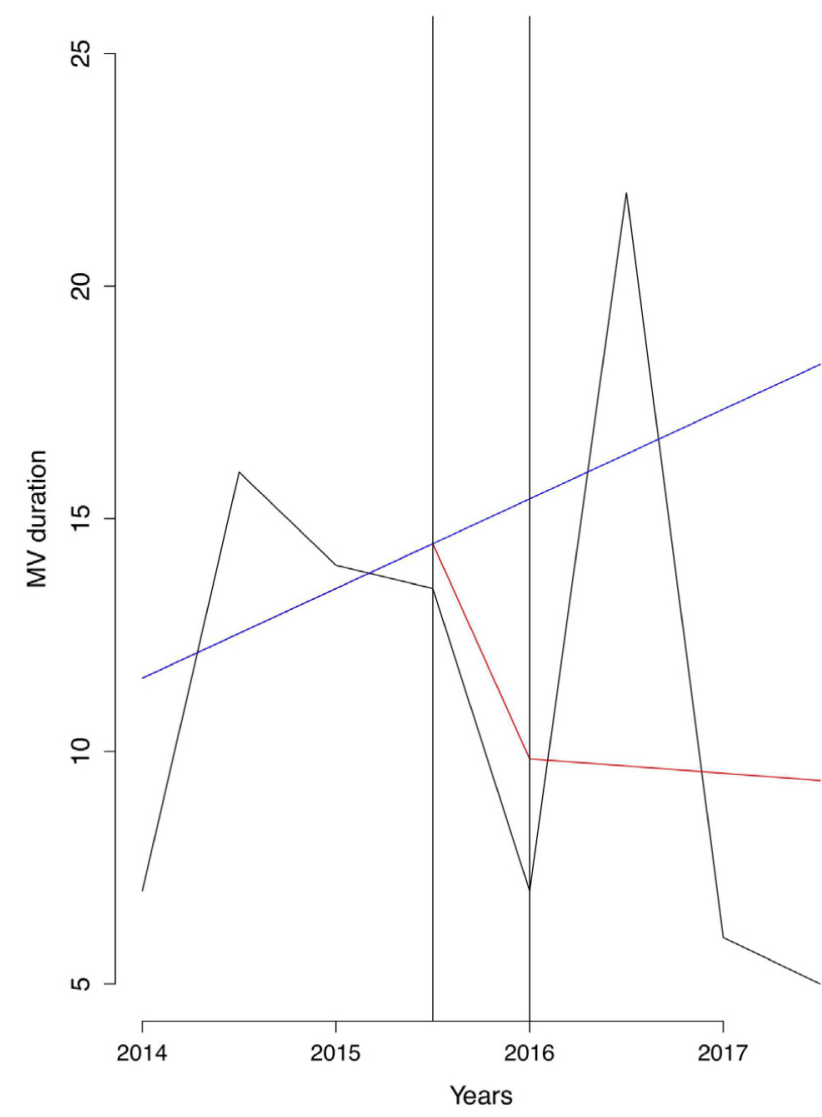

Fig. 2 - Time-interrupted time-series analysis of the aggregated mechanical ventilation duration between the Control and the Intervention Phases.

The figure displays the evolution of the aggregated value of mechanical ventilation duration during the study period. The aggregated value is the mean of the mechanical ventilation duration per semester. The vertical lines stand for the period of the implementation of the protocol. The lines embody the regression slope of the aggregated value. The blue regression line during the Intervention phase, embodies the estimated trend of aggregated value after 2016, if the sedation-analgesia protocol would not have been implemented. In the Control phase, an upward trend was revealed ( $\beta$ coefficient $1.25 \mathrm{CI}_{95}$ $[-2.3 ; 4.8])$, whereas the Intervention phase revealed a downward trend $\left(\beta\right.$ coefficient $\left.-0.5 \mathrm{CI}_{95}[-2.1 ; 1.1]\right)$. The difference between the two $\beta$ coefficients are not statistically different $(P=0.6)$. MV: mechanical ventilation.

some specific subsets such as burn patients lack guidelines on this topic. They are often excluded from these studies [4] owing to specific sedation-analgesia management secondary to burn care. Patients with major burn surface area usually undergo prolonged general anesthesia until complete skin graft recovery because of iterative surgery, potentially painful dressing changes and pain due to the burn(s). Such a strategy is not evidence-based and could lead to major side effects because of prolonged drug infusion. In a recent European survey performed in 188 specialized burn centers, Trupkovic et al. [18] evaluated the management of sedation and analgesia in burn patients. Less than a third of centers answered the
OR Spontaneous Breathing

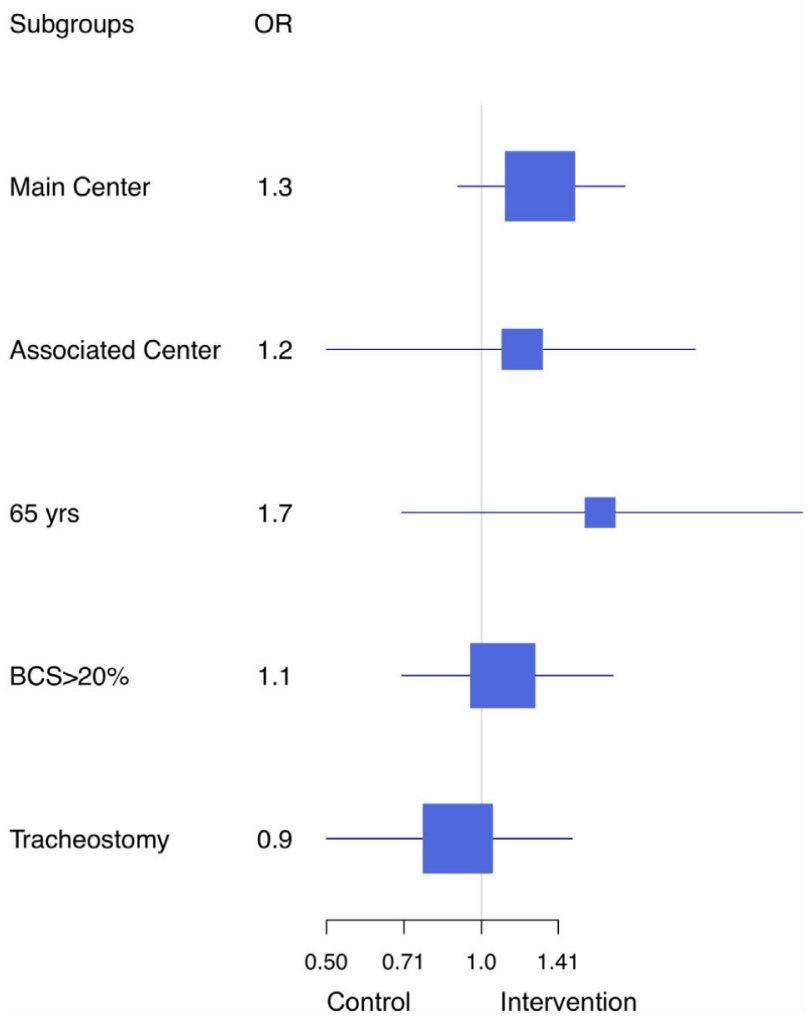

Fig. 3 - Forest plot regarding the odds ratio of extubation in subgroups.

BCS: Burn Cutaneous Surface.

survey and roughly $60 \%$ used sedation and analgesia scales routinely. The lack of evidence and guidelines was advocated to explain such a low rate of sedation-analgesia monitoring. We therefore provide our experience following implementation of a RASS/BPS protocol for the management of hypnotics and opioids in severe burn patients. Although this protocol was not associated with a significant decrease in the duration of mechanical ventilation, the management of sedation-analgesia with clinical scales is feasible in burn patients without side-effects and could be optimized to provide a positive impact on the outcome. Other strategies such as no-awakening trials or sedation holidays were not performed during the intervention phase, but could increase the impact of a sedation-analgesia protocol.

Low adherence remains in quality improvement projects. In a recent nationwide study in neuro-ICU patients [19], only $15 \%$ of the patients received care in full compliance with the protocol 4 months after its implementation. In a before-after study in patients with spinal trauma, only $8.3 \%$ of the patients received the full set of recommendations [15]. Indeed, the 
Table 2 - In-ICU management and outcome.

\begin{tabular}{|c|c|c|c|}
\hline & $\begin{array}{c}\text { Control phase } \\
\qquad \mathrm{N}=87\end{array}$ & $\begin{array}{l}\text { Intervention phase } \\
\qquad \mathrm{N}=101\end{array}$ & $P$ value \\
\hline VAP & $49(56.3)$ & $47(47)$ & 0.2 \\
\hline ARDS & $21(24.1)$ & $21(21)$ & 0.6 \\
\hline Catecholamine (days) & $4[0-12]$ & $3[0-11]$ & 0.8 \\
\hline Hypnotics (days) & $8[2-24]$ & $6[2-17]$ & 0.3 \\
\hline Opioids (days) & $17[4-32]$ & $8[3-23]$ & 0.06 \\
\hline Tracheostomy & $36(41.4)$ & $42(41.6)$ & 0.9 \\
\hline Surgery for burn care $(\mathrm{N})$ & $2[1-4]$ & $2[1-4]$ & 0.9 \\
\hline MV duration & $14[3-29]$ & $7[2-24]$ & 0.4 \\
\hline Day 90-VFD & 69 [31-87] & $2[33-88]$ & 0.3 \\
\hline ICU LOS & $25[9-45]$ & $24[10-44]$ & 0.7 \\
\hline Hospital LOS & $32[17-48]$ & $30[17-51]$ & 0.8 \\
\hline WLST & $9(10.3)$ & 12 (11.9) & 0.7 \\
\hline In-ICU mortality & $18(20.7)$ & $18(18)$ & 0.6 \\
\hline Day-90 mortality & $19(21.84)$ & $22(21.8)$ & 0.9 \\
\hline
\end{tabular}

Legend: VAP: Ventilator Acquired Pneumonia. ARDS: Acute Respiratory Distress Syndrome. MV: Mechanical Ventilation. ICU: Intensive Care Unit. LOS: Length of Stay. WLST: Withdrawal of Life Sustaining Therapies. VFD: Ventilatory Free Days.

successful implementation of guidelines is a slow process that depends on several factors including the methods of developing, disseminating, and implementing those recommendations. In our unit, the implementation of this protocol was associated with extra paramedic workload, no modification in the doses of sedation-analgesia during burn dressing, which could have hampered the beneficial effects of the protocol. Moreover, other strategies such as daily interruption [20] which could have been helpful, were not used. These aspects could explain the disappointing results in the reduction of the duration in mechanical ventilation. Some specific tools have been elaborated such as the Consolidated Framework for Implementation Research in order to study and counteract these barriers [21]. This framework explores five major domains: intervention characteristics, inner settings, outer settings, characteristics of the individuals involved and the process of implementation. It provides a pragmatic structure for approaching complex, multidisciplinary levels, by embracing, consolidating, and unifying key constructs from published implementation theories. This framework has been used in a retrospective fashion in the ICU [22], and was used to identify the barriers to early patient mobilization. Before the implementation of a new protocol or during its process [23], the Consolidated Framework for Implementation Research could be efficient in helping medical and paramedical staff to improve implementation of the protocol, and thus its effects.

As with any study, our study has limitations. The beforeafter study design supports association but not causation. However, a before-after design avoids cross-contamination, which would have been complex to manage in a randomizedcontrolled trial. Moreover, before-after studies provide a good level of evidence [24] with an acceptable estimation of the effect of the intervention [25]. Owing to technical issues, we did not collect the mean daily doses of analgesic and sedative drugs before and after the intervention. This information would have been useful to demonstrate the application of the intervention. However, we observed an application rate above $90 \%$. It is also possible that our study lacks the power to demonstrate a potential beneficial effect of a sedation-analgesia protocol based on the RASS and BPS scales. In our unit, MV weaning and use of tracheostomy were protocolized. Thus, the extrapolation of our results in the ICU with other standards of care such as a no tracheostomy policy or no standardized protocol for mechanical ventilation weaning remains to be demonstrated. During the intervention period, we used multimodal analgesia with ketamine. This could have altered the effects of the analgesia-sedation protocol. However, owing to the prolonged infusion of opioids and hypnotics, we believe that the effects of this multimodal analgesia are minimal. It is also possible that our study lacks the power to demonstrate a potential beneficial effect of a sedation-analgesia protocol based on the RASS and BPS scales. This advocates for the urgent development of large multicenter, potentially international, research networks in critically burn patients.

\section{Conclusion}

The implementation of a sedation-analgesia protocol based on the evaluation of the RASS/BPS to adapt the continuous infusions of sedative and analgesics and the use of short duration general anesthesia for procedural pain in severe critical burn patients is feasible but is not associated with a decrease in the duration of invasive MV.

\section{Conflict of interest}

Pr Karim Asehnoune received fees from Baxter, Edwards, LFB, Fisher and Payckel. Pr Roquilly received fees from MSD and Biomérieux. Pr Jung received fees from Medtronic and Sedena Medical. Pr Jung received grants from the French Ministry of Health outside the scope of this article. 
[14] Roquilly A, Cinotti R, Jaber S, Vourc'h M, Pengam F, Mahe PJ, et al. Implementation of an evidence-based extubation readiness bundle in 499 brain-injured patients. a before-after evaluation of a quality improvement project. Am J Respir Crit Care Med 2013;188:958-66, doi:http://dx.doi.org/10.1164/ rccm.201301-01160C.

[1] Snell JA, Loh NHW, Mahambrey T, Shokrollahi K. Clinical review: the critical care management of the burn patient. Crit Care 2013;17:241, doi:http://dx.doi.org/10.1186/cc12706.

[2] Chung KK, Wolf SE, Renz EM, Allan PF, Aden JK, Merrill GA, et al. High-frequency percussive ventilation and low tidal volume ventilation in burns: a randomized controlled trial. Crit Care Med 2010;38:1970-7, doi:http://dx.doi.org/10.1097/ CCM.0b013e3181eb9d0b.

[3] Barr J, Fraser GL, Puntillo K, Ely EW, Gélinas C, Dasta JF, et al. Clinical practice guidelines for the management of pain, agitation, and delirium in adult patients in the intensive care unit. Crit Care Med 2013;41:263-306, doi:http://dx.doi.org/ 10.1097/CCM.0b013e3182783b72.

[4] Riker RR, Shehabi Y, Bokesch PM, Ceraso D, Wisemandle W, Koura F, et al. Dexmedetomidine vs midazolam for sedation of critically ill patients: a randomized trial. Jama 2009;301:489-99, doi:http://dx.doi.org/10.1001/jama.2009.56.

[5] Shehabi Y, Bellomo R, Reade MC, Bailey M, Bass F, Howe B, et al. Early intensive care sedation predicts long-term mortality in ventilated critically ill patients. Am J Respir Crit Care Med 2012;186:724-31, doi:http://dx.doi.org/10.1164/rccm.20120305220C.

[6] Chanques G, Jaber S, Barbotte E, Violet S, Sebbane M, Perrigault PF, et al. Impact of systematic evaluation of pain and agitation in an intensive care unit. Crit Care Med 2006;34:1691-9, doi:http://dx.doi.org/10.1097/01. CCM.0000218416.62457.56.

[7] Chanques G, Conseil M, Roger C, Constantin JM, Prades A, Carr $\mathrm{J}$, et al. Immediate interruption of sedation compared with usual sedation care in critically ill postoperative patients (SOSVentilation): a randomised, parallel-group clinical trial. Lancet Respir Med 2017;5:795-805, doi:http://dx.doi.org/10.1016/ S2213-2600(17)30304-1.

[8] Janzekovic Z. A new concept in the early excision and immediate grafting of burns. J Trauma Inj Infect Crit Care 1970;10:1103-8.

[9] Gacto-Sanchez P. Surgical treatment and management of the severely burn patient: review and update. Med Intensiva 2017;41:356-64, doi:http://dx.doi.org/10.1016/j. medin.2017.02.008.

[10] Cancio LC, Salinas J, Kramer GC. Protocolized Resuscitation of Burn Patients. Crit Care Clin 2016;32:599-610, doi:http://dx. doi.org/10.1016/j.ccc.2016.06.008.

[11] Le Floch R, Naux E, Pilorget A, Arnould JF. Use of remifentanil for analgesia during dressing change in spontaneously breathing non-intubated burn patients. Ann Burns Fire Disasters 2006;19:136-9.

[12] Asehnoune K, Seguin P, Lasocki S, Roquilly A, Delater A, Gros A, et al. Extubation success prediction in a multicentric cohort of patients with severe brain injury. Anesthesiology 2017;127:338-46, doi:http://dx.doi.org/10.1097/ ALN.0000000000001725.

[13] Roquilly A, Cinotti R, Jaber S, Vourc'h M, Pengam F, Mahe PJ, et al. Implementation of an evidence-based extubation readiness bundle in 499 brain-injured patients. A before-After evaluation of a quality improvement project. Am J Respir Crit Care Med 2013;188:958-66, doi:http://dx.doi.org/10.1164/ rccm.201301-01160C.

[15] Cinotti R, Demeure dit latte D, Mahe PJ, Langlais P, Grillot N, Bouras $\mathrm{M}$, et al. Impact of a quality improvement program on the neurological outcome of patients with traumatic spinal cord injury: a before-after mono-centric study. J Neurotrauma 2019, doi:http://dx.doi.org/10.1089/neu.2018.6298 neu.2018.6298.

[16] Bodet-Contentin L, Frasca D, Tavernier E, Feuillet F, Foucher Y, Giraudeau B. Ventilator-free day outcomes can Be misleading. Crit Care Med 2018;46:425-9, doi:http://dx.doi.org/10.1097/ CCM. 0000000000002890 .

[17] Minhas MA, Velasquez AG, Kaul A, Salinas PD, Celi LA. Effect of protocolized sedation on clinical outcomes in mechanically ventilated intensive care unit patients: a systematic review and meta-analysis of randomized controlled trials. Mayo Clin Proc 2015;90:613-23, doi:http://dx.doi.org/10.1016/j. mayocp.2015.02.016.

[18] Trupkovic T, Kinn M, Kleinschmidt S. Analgesia and sedation in the intensive care of burn patients: results of a European survey. J Intensive Care Med 2011;26:397-407, doi:http://dx. doi.org/10.1177/0885066610393442.

[19] The BI-VILI study group, Asehnoune K, Mrozek S, Perrigault PF, Seguin P, Dahyot-Fizelier C, et al. A multi-faceted strategy to reduce ventilation-associated mortality in brain-injured patients. The BI-VILI project: a nationwide quality improvement project. Intensive Care Med 2017287:, doi:http:// dx.doi.org/10.1007/s00134-017-4764-6 345-314.

[20] Kress JP, Pohlman AS, O'Connor MF, Hall JB. Daily interruption of sedative infusions in critically ill patients undergoing mechanical ventilation. N Engl J Med 2000;342:1471-7, doi: http://dx.doi.org/10.1056/NEJM200005183422002.

[21] Damschroder LJ, Aron DC, Keith RE, Kirsh SR, Alexander JA, Lowery JC. Fostering implementation of health services research findings into practice: a consolidated framework for advancing implementation science. Implement Sci 2009;4: 886-915, doi:http://dx.doi.org/10.1186/1748-5908-4-50

[22] Costa DK, White MR, Ginier E, Manojlovich M, Govindan S, Iwashyna TJ, et al. Identifying barriers to delivering the awakening and breathing coordination, delirium, and early Exercise/Mobility bundle to minimize adverse outcomes for mechanically ventilated patients. Chest 2017;152:304-11, doi: http://dx.doi.org/10.1016/j.chest.2017.03.054.

[23] Balas MC, Burke WJ, Gannon D, Cohen MZ, Colburn L, Bevil C, et al. Implementing the awakening and breathing coordination, delirium Monitoring/Management, and early Exercise/Mobility bundle into everyday care. Crit Care Med 2013;41:S116-27, doi:http://dx.doi.org/10.1097/ CCM.0b013e3182a17064

[24] Barton S. Which clinical studies provide the best evidence? The best RCT still trumps the best observational study. Bmj 2000;321:255-6.

[25] Concato J, Shah N, Horwitz RI. Randomized, controlled trials, observational studies, and the hierarchy of research designs. N Engl J Med 2000;342:1887-92, doi:http://dx.doi.org/10.1056/ NEJM200006223422507. 